\title{
Manejo y tratamiento microquirúrgico de las dilataciones infundibulares de la arteria comunicante posterior. Serie de nueve casos y revisión de la literatura
}

\author{
J. M. González-Darder; F. Verdú-López y V. Quilis-Quesada
}

Servicio de Neurocirugía. Hospital Clínico Universitario de Valencia. Servicio Valenciano de Salud

Resumen

Introducción. La dilatación infundibular (DI) de la arteria comunicante posterior (AComP) se define como la dilatación cónica, triangular o en forma de embudo, menor de $3 \mathrm{~mm}$, en el origen de la AComP de la arteria carótida interna. El propósito del presente trabajo es presentar la experiencia propia en el tratamiento microquirúrgico de la DI de la AComP, revisar la bibliografía y proponer algoritmos para optimizar su manejo clínico y microquirúrgico.

Material y métodos. Se han estudiado nueve casos de DI intervenidos a través de un abordaje pterional. En cuatro pacientes con hemorragia subaracnoidea (HSA) la DI se consideró la única causa del sangrado; en otros cuatro pacientes la DI se intervino tras una HSA por ruptura de otra lesión aneurismática; finalmente, en un paciente con pseudoxantoma elástico la DI se intervino de forma preventiva.

Resultados. En ocho casos se procedió al clipaje de la DI y cierre de la AComP y en uno al reforzamiento de la DI al tratarse de una AComP del tipo fetal. No aparecieron complicaciones salvo una paresia transitoria del III par. El Glasgow Outcome Scale al alta y al año fue de 5 en todos los casos.

Conclusiones. La DI de la AComP es una lesión de significado no aclarado, pero que plantea la necesidad de considerar su tratamiento en algunas ocasiones: 1 . En pacientes con aneurismas rotos sometidos a cirugía y DI homolateral se recomienda explorar y tratar la lesión; 2. En pacientes con aneurismas rotos tratados con embolización con DI no tratada y en aquellos tratados con microcirugía con una DI contralateral, se considerarán en cada caso los factores de riesgo para indicar un tratamiento microquirúrgico; 3. En los pacientes con DI como única lesión vascular asociada a una HSA debe repetirse la angiografía y, en ausencia de otra explicación del sangrado, considerar el tratamiento microquirúrgico; 4 . El hallazgo de una DI en

Recibido: 3-10-10. Aceptado: 9-08-10 pacientes sin sangrado es una indicación de observación con neuroimagen y control de los factores generales de riesgo vascular, en ausencia de sustanciales factores de riesgo.

PALABRAS CLAVE: Dilatación infundibular. Arteria comunicante posterior. Aneurisma cerebral. Hemorragia subaracnoidea. Craneotomía.

Management and microsurgical treatment of infundibular dilatations of the posterior communicating artery. Series of nine cases and review of the literature

\section{Summary}

Introduction. The infundibular dilatation (ID) of the posterior communicating (PCom) artery is defined as the conic, triangular or infundibular shaped, less than $3 \mathrm{~mm}$ wide, origin of the PCom artery from the internal carotid artery. The purpose of this paper is to present the personal experience in the microsurgical management of the ID, to review the literature and to propose some algorithms to improve its clinical and microsurgical management.

Material and methods. Nine cases of ID have been operated on through a pterional approach. In four patients with subarachnoid hemorrhage (SAH) the ID was considered as the potential source of the bleeding; in four cases the ID was treated after a SAH due to the rupture of an aneurysm; finally, an ID was treated in patient with diagnosis of pseudoxantoma elasticum.

Results. In eight cases the ID was clipped and the Pcom artery subsequently occluded and in the remaining case the ID was associated with a fetal PComA and the ID was reinforced. There were no complications

Abreviaturas. ACI: arteria carótida interna. AComP: arteria comunicante posterior. ACP: arteria cerebral posterior. DI: dilatación infundibular. HSA: hemorragia subaracnoidea. ID: infundibular dilatation. PCom: posterior communicating. SAH: subarachnoid hemorrhage. 
excepting a transitory third cranial nerve paresis. The Glasgow Outcome Scale was 5 in all cases at discharge and one year later.

Conclusions. The true significance of the ID remains unknown, but in some instances it is necessary to consider its management: 1. In patients with ruptured aneurysms submited to microsurgical clipping and with an ipsilateral ID, the lesion must be explored and treated; 2 . In patients with ruptured aneurysms treated with endovascular procedures or harbouring an ID contralateral to a microsurgically treated aneurysm, the microsurgical indication will be done after considering all risk factors; 3 . In patients with $\mathrm{SAH}$ and an ID as the only potential source of the bleeding there would be an indication for microsurgical exploration; 4 . The incidental finding of an ID should be indication for observation in absence of major risk factors.

KEY WORDS. Infundibular dilatation. Posterior communicating artery. Cerebral aneurysm. Subarachnoid hemorrhage. Craniotomy.

\section{Introducción}

La dilatación infundibular (DI) de la arteria comunicante posterior (AComP) se define como la dilatación con forma cónica, triangular o de embudo, menor de $3 \mathrm{~mm}$, en el origen de la AComP de la arteria carótida interna (ACI). La importancia de esta lesión es que continúa sin estar inequívocamente determinado si se trata de una variante anatómica, de una lesión preaneurismática o si puede ser causa de sangrado. Tampoco la DI es una lesión claramente definida desde el punto de vista histológico, anatómico, fisiopatológico, en los estudios de imagen ni tampoco en su pronóstico, aunque en la mayor parte de los casos evoluciona favorablemente. Todos estos aspectos y sus correspondientes implicaciones clínicas deben considerarse a la hora de manejar pacientes con lesiones de pequeño tamaño en la salida de la AComP con o sin hemorragia subaracnoidea (HSA), donde se plantea el diagnóstico diferencial entre una DI verdadera, una lesión preaneurismática o un aneurisma de pequeño tamaño. El propósito del presente trabajo es presentar la experiencia propia en el tratamiento de estas lesiones, revisar de forma exhaustiva la bibliografía reciente sobre el tema y proponer unos algoritmos para optimizar su manejo clínico y quirúrgico.

\section{Material y métodos}

En el estudio se incluyen los casos de DI de la AComP demostrados quirúrgicamente de la serie de lesiones aneurismáticas intracraneales tratadas quirúrgicamente por el primer autor entre los años 1996-2006. Los datos epidemiológicos, de imagen y evolutivos se recogieron de forma prospectiva en una base de datos de la que se han obtenido los parámetros estudiados. Se revisaron también las imágenes fotográficas y de vídeo registradas en las cirugías así como los esquemas quirúrgicos incluidos en las hojas operatorias.

Los criterios para considerar una lesión como DI en los estudios de imagen incluían una lesión cónica, triangular o en embudo, de pequeño tamaño, simétrica y regular, en la salida de la AComP en la angiografía lateral estricta (fig. 1). En la exploración quirúrgica se contemplaban los mismos

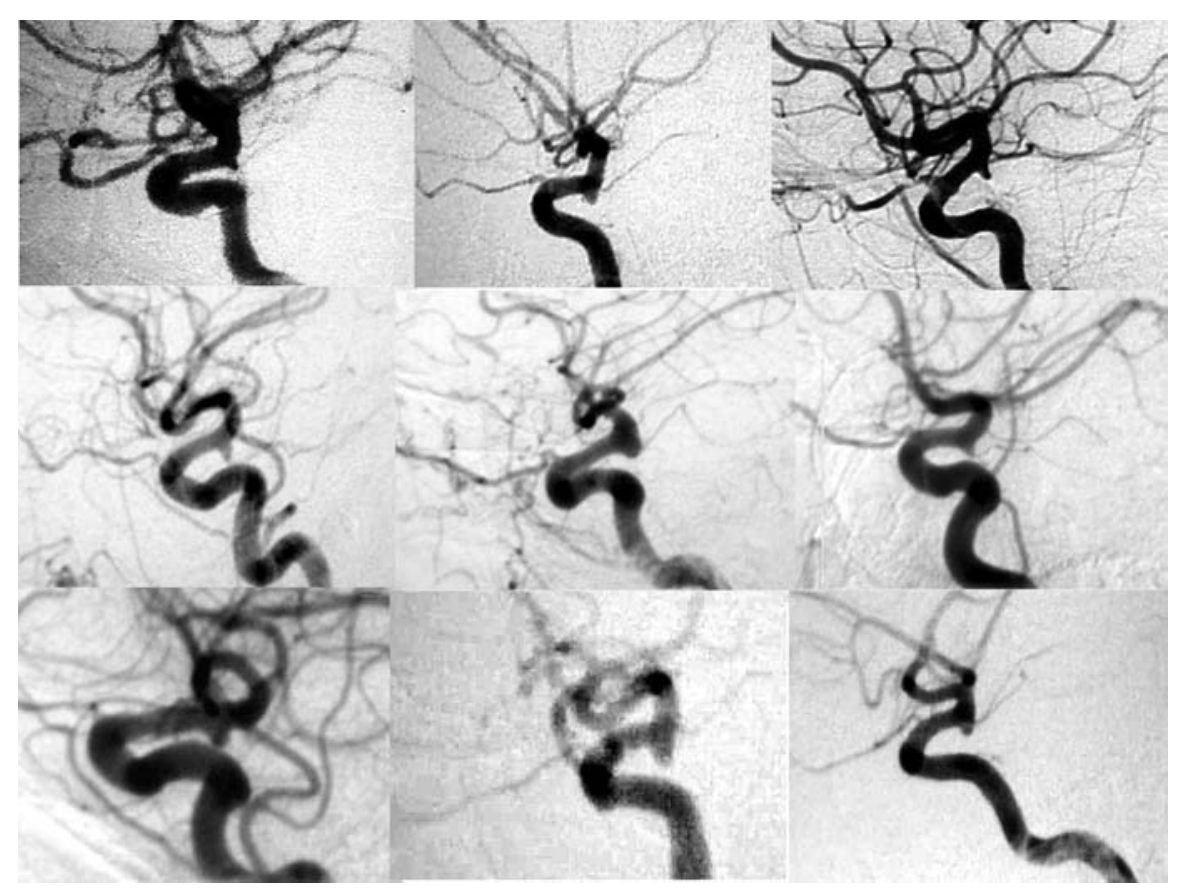

Figura 1. Estudio angiográfico en proyección lateral de los pacientes incluidos en la serie (orientadas todas en el mismo sentido). 


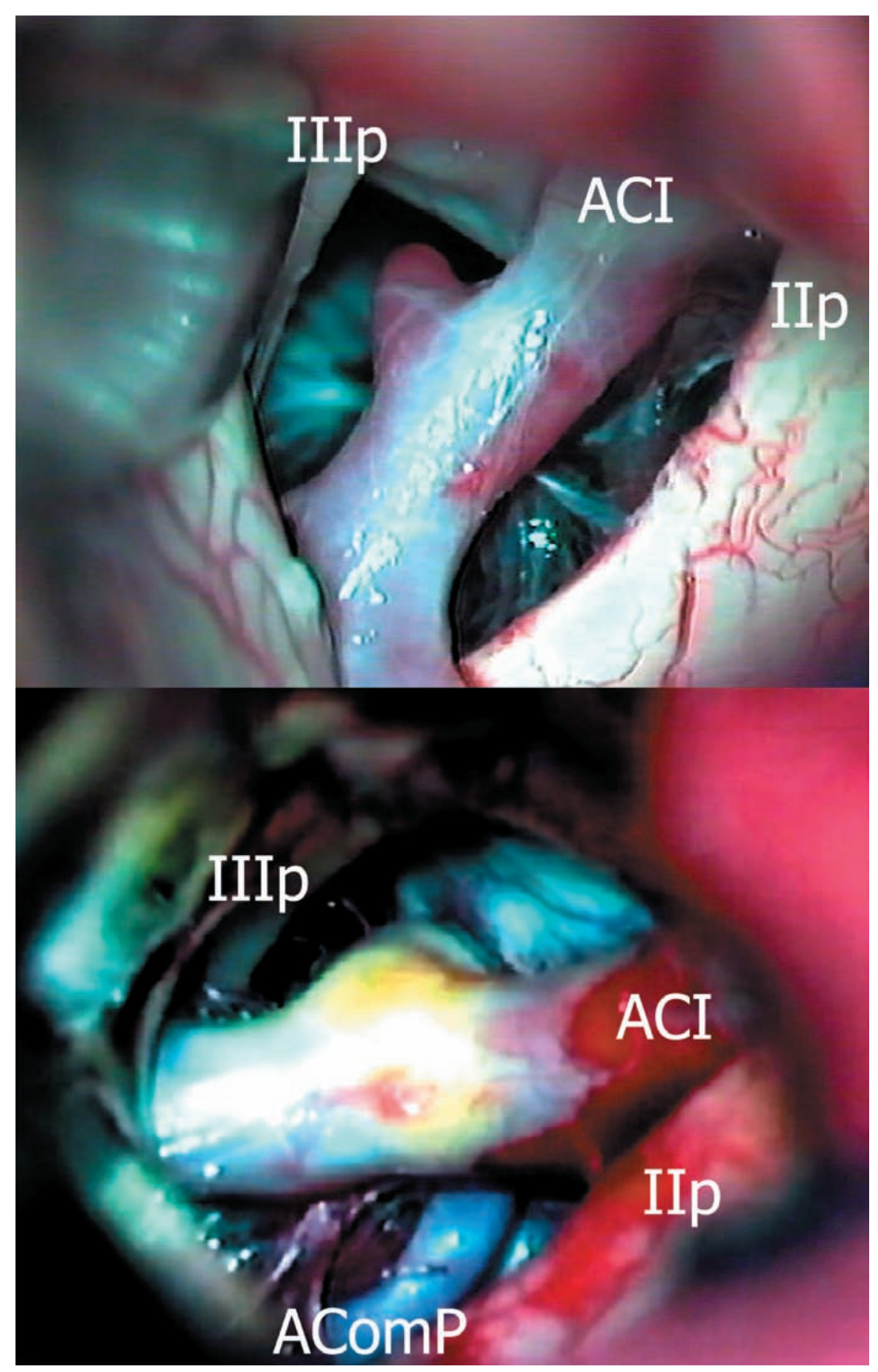

Figura 2. Imágenes quirúrgicas del abordaje microquirúrgico a través de un abordaje pterional izquierdo: Arriba: DI con pared adelgazada y transparente y AComP del tipo 'adulto'. Abajo: DI con aspecto normal de la pared y AComP del tipo 'fetal'. (IIp: nervio óptico; IIIp: nervio motor ocular común; ACI: arteria carótida interna, AComP: arteria comunicante posterior).

criterios, pero había que demostrar la salida de la AComP del vértice de la lesión. En todos los casos, tanto en la angiografía como en la exploración quirúrgica, se clasificó la AComP como del tipo 'fetal', si su diámetro era igual o mayor al del segmento P1 de la arteria cerebral posterior (ACP) homolateral, o del tipo 'adulto', en caso contrario $^{22,29}$. En todos los casos se describe el aspecto externo de la lesión (regular o/irregular; pared vascular normal, trans- 
González-Darder y col

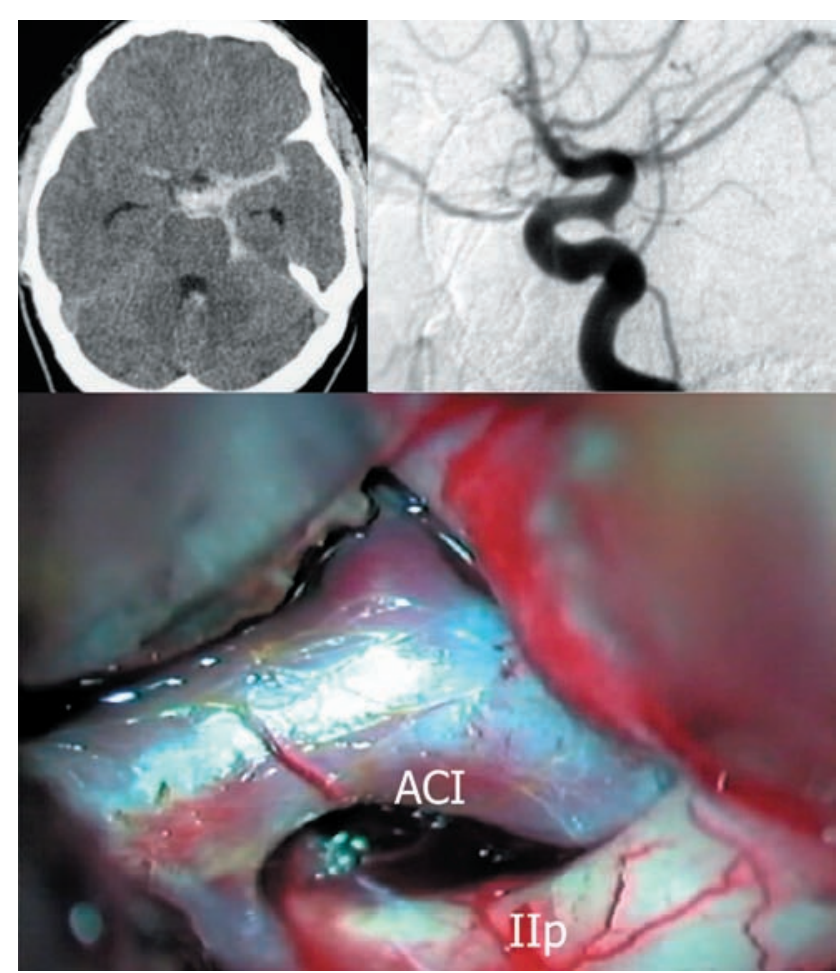

Figura 3. DI izquierda en un paciente con hemorragia subaracnoidea en cisternas basales, de predominio izquierdo (arriba izquierda). Angiografía de la única lesión encontrada en la angiografía, típica de DI, con salida de la comunicante posterior del extremo de la misma (arriba derecha). Abajo: Imagen quirúrgica a través de un abordaje pterional donde se aprecia la lesión, con paredes adelgazadas, debajo del pliegue petroclinoideo. (IIp: nervio óptico; ACI: arteria carótida interna).

parente o con abultamientos). Todos los pacientes fueron intervenidos a través de un abordaje pterional homolateral y utilizando técnicas microquirúrgicas standard (fig. 2). Se exponía tanto la lesión como todo el trayecto de la AComP desde su salida de la ACI hasta la ACP, identificando todas sus las arterias perforantes.

\section{Resultados}

Se incluyen en el estudio un total de 9 pacientes, lo que supone el $2.8 \%$ de la casuística de aneurismas cerebrales rotos y no rotos intervenidos quirúrgicamente en el periodo de estudio. Cuatro pacientes eran hombres y 5 mujeres, con una edad media de 47.9 años (rango 31-60). Cuatro pacientes habían sufrido una HSA atribuida a la lesión al no haber otros hallazgos en el estudio vascular (fig. 3), aunque de escasa repercusión clínica (WFNS grado 1 en 3 pacientes y grado 2 en 1 paciente ${ }^{21}$; Fisher grado 0 en 2 pacientes diagnosticados con punción lumbar y Fisher grado 1 en 2 pacientes). En cinco pacientes la lesión no había san-
Neurocirugía

2011; 22: 301-309

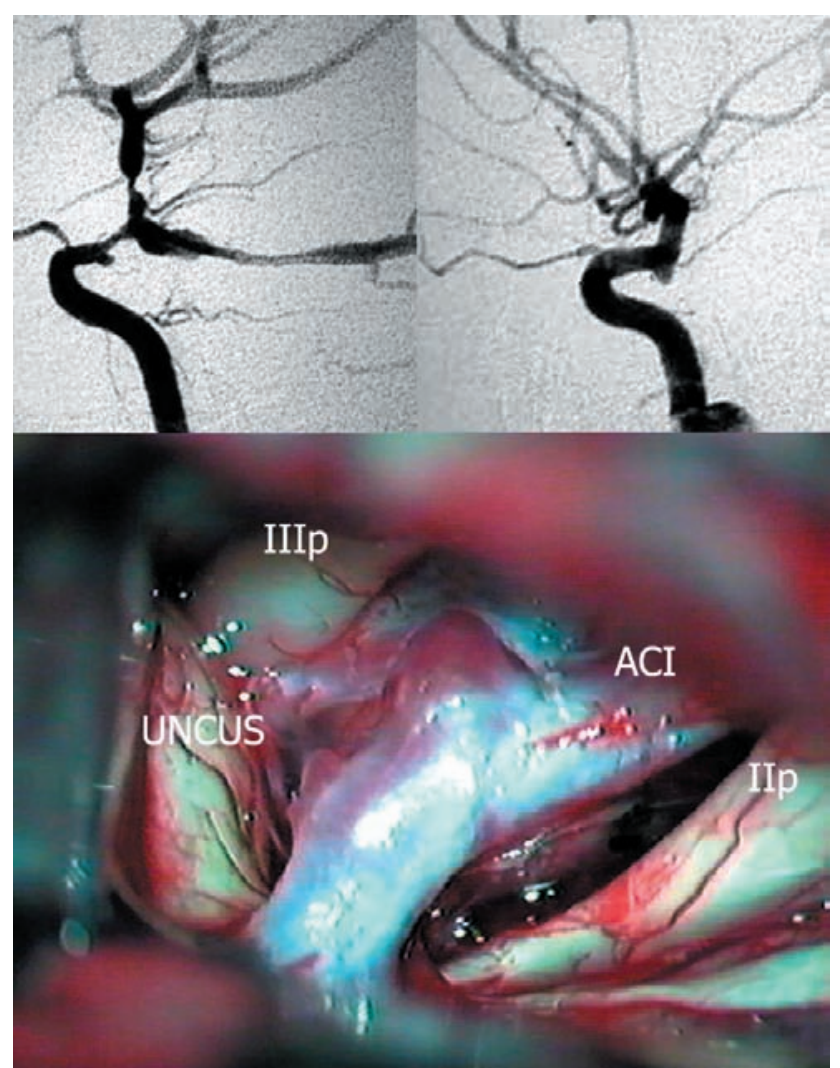

Figura 4. DI izquierda en una paciente con aneurisma roto de AComP derecha tratado previamente con clipaje microquirúrgico. Arriba izquierda: angiografía del aneurisma de AComP derecho roto, con severo vasoespasmo regional y AComP de tipo fetal. Arriba derecha: Angiografía de la lesión izquierda, sin representación de la AComP pero con relleno de la arteria coroidea anterior. Abajo: Imagen quirúrgica a través de un abordaje pterional donde se aprecia la DI, con pared adelgazada y que se continua con una AComP del tipo 'adulto' (flecha), y sus relaciones con el III par. Distalmente a la DI se aprecia la salida de la arteria coroidea anterior, también de aspecto infundibular. (IIp: nervio óptico; IIIp: nervio motor ocular común; ACI: arteria carótida interna; UNCUS: uncus del lóbulo temporal).

grado y fue identificada tras estudio vascular. Uno de ellos había sido estudiado por pseudoxantoma elástico y en los otros cuatro pacientes se identificó la DI tras una HSA aneurismática por rotura de un aneurisma contralateral de AComP (3 pacientes) (fig. 4) y de arteria cerebral media (1 paciente), todos ellos excluidos quirúrgicamente. Cinco lesiones estaban en el lado izquierdo y las cuatro restantes en el lado derecho. Además de las DI intervenidas, los pacientes tenían otros seis aneurismas, los cuatro rotos ya referidos, y otros dos no rotos. Un paciente asociaba una malformación arteriovenosa temporal homolateral, que fue intervenida simultáneamente. El diagnóstico se realizó en 
todos los casos con angiografía, aunque en algunos pacientes se asoció angio-TAC y/o angio-RNM con reconstrucción tridimensional. La AComP sólo fue visible en los estudios angiográficos en tres casos, siempre como tipo del 'adulto' (fig. 1).

Todos los pacientes fueron intervenidos por un abordaje pterional, exponiendo siempre la AComP en toda su extensión. Durante la exploración microquirúrgica la AComP fue clasificada como del tipo 'fetal' en un caso y del 'adulto' en el resto (fig. 2). En seis de los casos, la pared de la DI presentaba un aspecto adelgazado y transparente, pero sin encontrar abultamientos o lesiones aneurismáticas, mientras que en los tres casos restantes la pared de la DI tenía aspecto de pared vascular normal (fig. 2, 3 y 4). No se identificaron signos directos de rotura de la lesión en ninguno de los casos. En el caso de DI con arterias del tipo 'adulto', se procedía a la colocación de uno o dos clips excluyendo la DI y la AComP hasta la salida de las primeras perforantes. En el único caso de arteria del tipo 'fetal' se realizó el refuerzo de la pared de la DI con músculo y material hemostático. No aparecieron complicaciones postoperatorias relevantes, salvo una paresia transitoria de III par. En cuatro pacientes se realizaron angiografías de control, con exclusión en todos los casos de las lesiones intervenidas. El Glasgow Outcome Score ${ }^{12}$ al alta era de 5 en todos los casos y se mantuvo estable después de un año de evolución.

\section{Discusión}

\section{Dilatación infundibular de la arteria comunicante pos- terior}

Desde un punto de vista conceptual ${ }^{16,25}$, la dilatación infundibular (DI) de la AComP, también llamada infundíbulo o implante infundibular (del latín infundibulum: embudo), se define como una dilatación arterial de forma cónica, triangular o de embudo que puede aparecer en el origen de la AComP de la ACI. Se considera que la base, boca o porción más ancha del infundíbulo, que está en contacto con la ACI, debe tener una amplitud inferior a $3 \mathrm{~mm}$, aunque en alguna series quirúrgicas se acepta un tamaño mayor ${ }^{7}$. La AComP debe emerger del vértice opuesto a la base de la lesión en la ACI. Por definición, las DI no tienen cuello y son simétricas o regulares, en contraposición con los aneurismas que suelen presentar un cuello más o menos bien definido y una morfología que puede ser asimétrica o un contorno irregular. La frecuencia de las DI recogida en la literatura varía ampliamente, dependiendo del método de estudio y población estudiada ${ }^{2}$. La DI de la AComP es la más frecuente a nivel de la vascularización intracraneal, pero puede aparecer en el origen de otras ramas o bifurcaciones de las arterias cerebrales como es el caso de la arte- ria coroidea anterior o de la cerebelosa posteroinferior. La evolución es generalmente benigna, pero en algunos casos puede romperse y sangrar y en otros casos se ha demostrado su evolución hacia un aneurisma de AComP.

Desde un punto de vista clínico es importante distinguir entre la DI verdadera y los aneurismas saculares verdaderos de pequeño tamaño, ya que cada tipo lesional tendría implicaciones diferentes. Sin embargo, además de estos dos tipos de lesión hay que considerar la llamada DI preaneurismática, definida por Endo et al. como aquella DI de tamaño grande que en la exploración quirúrgica presenta prominencias o bultos rojizos en su superficie, sugiriendo áreas débiles en la pared ${ }^{7}$. Así, estos autores encontraron que de 34 lesiones no rotas y mayores de $3 \mathrm{~mm}$ (diámetro medio de $4.9 \mathrm{~mm}$ ), consideradas angiográficamente aneurismas de la AComP, al ser exploradas quirúrgicamente sólo fueron confirmados como tales seis casos, 24 se clasificaron como DI verdaderas ya que no presentaban ninguna anormalidad en sus paredes y, finalmente, cuatro se consideraron DI preaneurismáticas ya que presentaban abultamientos en su cara lateral.

Teóricamente, la DI verdadera debería ser considerada como una variación de la normalidad sin un sustrato patológico en la pared arterial. Frente a ello, el aneurisma sacular verdadero, aunque sea de pequeño tamaño, tiene un evidente significado lesional y se asocia a típicos cambios anatomopatológicos de la pared arterial, que pueden ser más conspicuos en caso de crecimiento o ruptura. Sin embargo, existen pocos datos histopatológicos de las DI y los resultados son contradictorios al faltar estudios amplios y sistemáticos. Así, frente a los resultados de Epstein et al. ${ }^{8}$, quienes estudian siete DI no rotas encontradas en autopsias y concluyen indicando categóricamente que en todas ellas la morfología de la pared vascular era normal, Hassler y Saltzmann ${ }^{11}$ también estudiando siete casos no rotos de autopsia encontraron defectos en la capa media en siete y destrucción en la elástica interna en uno. En esta misma línea, se han descrito cambios murales en DI rotas. Archer et al. ${ }^{1}$ estudiaron una DI rota en la que encuentran pérdida y fragmentación de la capa elástica interna y una capa media presente pero fina y más recientemente, Oyhama et al. ${ }^{18}$ publican un caso de rotura fatal de una DI en la que el examen histológico demostraba la rotura de la lesión, que presentaba engrosamiento de la íntima y disección de la media, con rotura de los elementos musculares y elásticos en ambas zonas. La existencia de cambios en la pared semejantes a los encontrados en los aneurismas hizo suponer que la DI podría ser una lesión preaneurismática. Probablemente, sea la DI preaneurismática descrita por Endo et al. ${ }^{7}$, la que tendría cambios en la pared semejantes a los del aneurisma, aunque poco intensos, incompletos o más sutiles, mientras que la DI verdadera tenga una pared normal y los aneurismas muestren cambios mas graves, en especial si se han roto. 


\section{Diagnóstico por imagen}

Puede resultar difícil a veces establecer con la angiografía la verdadera naturaleza de una lesión ubicada en el origen de la AComP, sobre todo si es de pequeño tamaño o si la AComP no se rellena, y así distinguir entre la DI, verdadera o preaneurismática, y un aneurisma. El relleno de la AComP es crucial para calificar una dilatación como DI o aneurisma, pero sólo se visualiza en poco más de la mitad de los $\operatorname{casos}^{29}$. En nuestra serie de casos, la AComP sólo se rellena en la tercera parte de nuestros pacientes. En este sentido, los estudios iniciales ya reconocían esta dificultad y recomendaban la angiografía vertebral con compresión carotídea para favorecer el relleno de la AComP a contracorriente ${ }^{9}$. Una dificultad añadida es la posibilidad de superposición de un aneurisma con la AComP que produzca la falsa impresión diagnóstica de que esta emerja de una DI. Endo et al. ${ }^{7}$ correlacionaron el tipo de lesión con el patrón de relleno y grosor de la AComP, llamando la atención de que los aneurismas y DI preaneurismáticas se relacionan mas frecuentemente con una AComP del tipo 'fetal', mientras que las DI verdaderas se asociaban a una AComP del tipo 'adulto' o que no se rellenaba. Buscando signos diagnósticos específicos de la DI, Kaufman et al. ${ }^{13}$ describen el signo del 'hoyuelo' ('dimpled appearance') en las DI, que no es más que una radiolucencia redondeada en la DI durante la angiografía, habitualmente transitoria, debida al efecto de lavado de la sangre sin contraste procedente a contracorriente por la AComP desde el territorio vertebrobasilar. El uso de angio-TAC con reconstrucción en tres dimensiones es útil en el manejo de aneurismas de AComP ya que ofrece referencias importantes como los elementos óseos y otros vasos mostrando además su configuración espacial, pero no parece ser el más adecuado para el diagnóstico y seguimiento de las DI debido a su pequeño tamaño ${ }^{10,26}$. Un método no invasivo que puede ayudar a mejorar el diagnóstico de la DI o diferenciar ésta de un aneurisma puede ser la fusión de imágenes en tres dimensiones de cisternografía y angiografía obtenidas mediante RM lo que mejora la resolución obtenida por las técnicas y secuencias previas de RM utilizadas hasta ahora ${ }^{23}$. En todo caso, el diagnóstico por imagen de la DI presenta poca especificidad y muestra grandes contradicciones con los hallazgos operatorios, tanto en el aspecto de la lesión como en el tamaño relativo de la AComP y sus relaciones con la DI, como se demuestra en nuestro estudio.

\section{Aspectos clínicos}

La DI es una lesión asintomática, de buen pronóstico y que constituye habitualmente un hallazgo en los estudios vasculares de imagen. La trascendencia clínica de la lesión reside cuando se encuentra en el contexto de una HSA o a la hora de dar un consejo al paciente, en el que se ha encontrado casualmente, acerca de su posible evolución a un aneurisma. Como hemos indicado, los criterios de diagnóstico diferencial entre la DI verdadera y el aneurisma pueden estar a veces claros, pero las características definitivas de la lesión y su tipo sólo se identifica en la exploración quirúrgica.

Hay descritos casos en la literatura que demuestran la transformación de una DI de la AComP en un aneurisma de AComP y que éste puede romperse. La magnífica revisión de Marshman et al. ${ }^{16}$ recoge hasta 1998 un total de 11 casos de aneurismas de AComP desarrollados sobre DI conocidas en estudios previos. Desde entonces se han referido algunos casos adicionales. Así, se han descrito casos de rotura de un aneurisma de AComP desarrollado a partir de una DI identificada angiográficamente tras una HSA por rotura demostrada de un aneurisma por Cowan et $a .^{5}$ en un caso tras 5 años y por Yamakawa et al. ${ }^{28}$ en tres pacientes incluidos en una serie de 20 casos con resangrado tardío tras HSA. Martins et al. ${ }^{17}$ aportan un caso semejante tras 11 meses, pero en este caso la lesión había sido explorada quirúrgicamente demostrándose que era una DI verdadera que fue dejada sin tratar. También describen Radulovic et al. ${ }^{20}$ dos casos de desarrollo y ruptura de aneurismas de AComP sobre DI descubiertas 9 y 11 años antes tras una HSA cuyo único hallazgo fue la DI. Por su parte, Coupe et al. ${ }^{4}$ y Kuwahara et al. ${ }^{14}$ demuestran operatoriamente la ruptura de una DI identificada tras una HSA. Así pues, existen casos documentados en donde se demuestra la transformación de una DI en un aneurisma que se ha roto y otros casos en donde se ha producido la rotura de una DI originándose una HSA. Frente a todo lo expuesto, hay grandes series que no demuestran sangrado o transformación aneurismática de las DI. Así, los datos del estudio cooperativo publicado por Locksley ${ }^{15}$ no revelaron ningún caso de rotura de DI cuando ésta tenía un tamaño menor de $3 \mathrm{~mm}$ ni tampoco Wollschlaeger et al. ${ }^{27}$ encontraron ningún signo de ruptura en todas las DI identificadas en su extensa serie de autopsias.

\section{Aspectos fisiopatológicos}

Ante las evidencias clínicas de que, aunque sea excepcionalmente, una DI puede progresar hasta llegar a convertirse en un aneurisma, existe la posibilidad de que esta transformación sólo ocurra en aquellas DI preaneurismáticas que ya tengan cambios histológicos aneurismáticos, bien sean congénitos o adquiridos, hipótesis que se plantea en la propia génesis de los aneurismas saculares. Otra posibilidad es que la misma forma de la DI predisponga a la progresiva dilatación de la misma debido a su configuración hidrodinámica, ya que aplicando el principio de Bernoulli se demuestra que una súbita disminución 
de la velocidad al aumentar el diámetro resultaría en un aumento de la tensión de las paredes de la DI. También se han considerado factores hemodinámicos relacionados con el efecto 'jet' ${ }^{\prime 14}$. Ebina et al. ${ }^{6}$ estudiaron las características angiográficas de las DI y encontraron que una AComP bien desarrollada podría ser uno de los factores que puedan contribuir al desarrollo aneurismático a partir de una DI, en especial si ésta es grande o se asocia a un gran ángulo divergente entre la AComP y la ACI. La revisión de la bibliografía sugiere que existe una alta incidencia de buen llenado de la AComP en aneurismas desarrollados a partir de una DI. Sin embargo, como ocurre en el desarrollo de los aneurismas, pueden influir otros factores de riesgo sistémicos aparte de los murales y hemodinámicos. Así el paciente descrito por Patrick et al. ${ }^{19}$ había sido sometido a la ligadura de la arteria carótida contralateral, el de Kuwanara et al. ${ }^{14}$ sangró precozmente tras un by-pass extraintracraneal de arteria temporal superficial a cerebral media y el de Cowan et al. ${ }^{5}$ sufría la asociación del síndrome de Alagille y coartación de aorta, todos ellos procesos que aumentan el riesgo de desarrollo de aneurismas cerebrales. Los datos epidemiológicos recogidos de la literatura de los casos donde se ha demostrado la formación de un aneurisma a partir de una $\mathrm{DI}^{5,16,17,20}$ indican que esto ocurre más frecuentemente en mujeres, en pacientes jóvenes entre 30-40 años, en el lado izquierdo y en la mitad de los casos ante una DI bilateral. El tiempo medio de progresión es de unos 7 años (rango, 0,5-11 años), aunque el tiempo de progresión viene determinado por la fecha de realización de la prueba de imagen y esto hace que los datos no representen con exactitud la realidad. Ebina et al. ${ }^{6}$ en un interesante trabajo elaboraron una serie de conclusiones con implicaciones pronósticas: que las pequeñas DI tienden a crecer, y en su crecimiento pasan de una forma triangular a una forma redondeada; que las DI más grandes aparecen en pacientes jóvenes; que hay un mayor número de casos de DI en pacientes que tienen un aneurisma de la AComP contralateral; que la HTA, al igual que tener una AComP bien desarrollada, son factores predisponentes para tener una gran DI y de forma redondeada además en el segundo caso; que aumenta la posibilidad de tener una DI de tamaño intermedio o grande a mayor ángulo de divergencia formado entre la ACI y la AComP; y, finalmente, los autores proponían para todos estos casos un exhaustivo control y/o tratamiento agresivo.

\section{Tratamiento microquirúrgico}

Las características anatómicas de estas lesiones hacen habitualmente imposible su tratamiento endovascular, por lo que el manejo de la DI se hace siempre siguiendo criterios neuroquirúrgicos. En el diseño de las guías de actuación que proponemos a continuación hemos tratado de recoger todas las situaciones clínicas posibles y hemos tomado como elemento principal la seguridad del paciente protegiéndolo ante un eventual sangrado o resangrado, admitiendo que el diagnóstico seguro por imagen de la DI verdadera no es siempre posible. La primera situación sería la del paciente con un aneurismas roto programado para clipaje microquirúrgico y con una DI homolateral al aneurisma roto, lo que obliga a la exploración microquirúrgica de la lesión y a su eventual tratamiento en el mismo abordaje. En pacientes con HSA aneurismática sometidos a clipaje y con una DI contralateral hay que sopesar los factores de riesgo junto a la ansiedad del enfermo para recomendar observación con angiografías periódicas frente al tratamiento microquirúrgico preventivo de una eventual ruptura. Los factores de riesgo a considerar serían fundamentalmente de tipo personal (edad joven, hipertensión arterial, una historia familiar o personal de aneurismas, presencia de otros factores de riesgo vascular o enfermedades asociadas a aneurismas) o anatómico (aneurismas múltiples, bilateralidad de la DI, existencia de un aneurisma verdadero de la AComP contralateral, en especial si se ha roto, DI de un tamaño considerable $(>3 \mathrm{~mm})$ o con protuberancias asociadas, AComP del tipo fetal, un gran ángulo divergente entre la AComP y la ACI). Los pacientes en esta situación precisarían un nuevo abordaje pterional contralateral o bien pueden ser explorados de la DI contralateral a través del abordaje del lado de la lesión que va a ser clipada (craneotomía unilateral para aneurismas bilaterales) $)^{17}$. En este mismo grupo se incluirían también los pacientes con aneurismas rotos ya tratados con técnica endovascular y una DI homo o contralateral no tratada. Una tercera situación corresponde a los pacientes con HSA en donde la DI es la única lesión demostrada en los estudios de neuroimagen. En este caso, la recomendación sería repetir la angiografía y, en caso de persistir la DI como única etiología posible de la HSA, considerar el tratamiento microquirúrgico, especialmente si la HSA se acumula en las cisternas del lado de la $\mathrm{DI}^{4,13}$. En este sentido, Marshman et al. ${ }^{16}$ indican que no debería considerarse como 'negativa' una angiografía tras una HSA en la que se detecta una DI. Algunos autores proponen que debería plantearse un protocolo más conservador, con angiografías de control anuales para detectar eventuales cambios morfológicos de la lesión. Finalmente, tendríamos los pacientes sin HSA en los que la DI se descubre de forma accidental, donde la recomendación es la observación con imagen y control de los factores generales de riesgo vascular, salvo en presencia de factores de riesgo relevantes.

El tratamiento microquirúrgico se hace a través de un abordaje pterional clásico ${ }^{29}$, exponiendo todo el trayecto de la AComP desde su origen de la ACI hasta la ACP. La anatomía microquirúrgica de la AComP y de sus perforantes ha sido descrita con detalle y debe ser bien 
conocida en este tipo de abordaje, dada la gran cantidad de variaciones anatómicas ${ }^{3,32,29}$. En el caso de una arteria del tipo 'adulto' se procede a la exclusión de la DI con uno o dos clips colocados en la salida de la AComP de la ACI, proximales a las primeras perforantes. En caso de arteria del tipo 'fetal', dado que el flujo por la AComP se hace predominantemente desde la ACI, la exclusión de la arteria puede comprometer el flujo por las perforantes y por la propia ACP con peligro de déficits neurológicos. En este último caso se valora la angiografía, anatomía microquirúrgica, el flujo de la AComP y ACP con sonda microdoppler o evidencia de la permeabilidad de las perforantes con la microangiografía con fluorescencia ${ }^{24}$, decidiendo entonces entre el clipaje de la DI, la reconstrucción de la salida de la AComP o no realizar ninguna maniobra terapéutica, ya que el simple reforzamiento de la lesión es una maniobra que carece de eficacia demostrada previniendo el crecimiento o ulterior ruptura de aneurismas o DI y, además, puede dificultar la tarea de una eventual reparación quirúrgica ulterior de la lesión tratada de esta forma ${ }^{30}$.

\section{Conclusiones}

La DI de la AComP es una lesión de significado no aclarado, pero que plantea la necesidad de considerar su tratamiento microquirúrgico en algunas ocasiones. Para la toma de decisiones es necesario sopesar aspectos epidemiológicos, clínicos, anatómicos y hemodinámicos diferentes en cada uno de los escenarios. En pacientes con aneurismas rotos sometidos a cirugía y DI homolateral se recomienda explorar y tratar la lesión. En pacientes con aneurismas rotos tratados con embolización con DI no tratada y en aquellos tratados con microcirugía con una DI contralateral, se considerarán en cada caso los factores de riesgo para indicar un tratamiento microquirúrgico. En los pacientes con DI como única lesión vascular asociada a una HSA debe repetirse la angiografía y, en ausencia de otra explicación del sangrado, considerar la exploración microquirúrgica. Finalmente, el hallazgo de una DI en pacientes sin sangrado es una indicación habitual de observación con neuroimagen y control de los factores generales de riesgo vascular.

\section{Agradecimientos}

El primer autor quiere agradecer a todo el personal que ha colaborado en el diagnóstico y tratamiento de los pacientes incluidos en el estudio, en especial a los miembros de los Servicios de Neurocirugía del Hospital General de Castellón y del Hospital Clínico Universitario de Valencia.

\section{Bibliografía}

1. Archer, C.R., Silbert, S.: Infundibula may be clinically significant. Neuroradiology 1978; 15: 247-251.

2. Avci, E., Bademci, G., Oztürk, A.: Posterior communicating artery: from microsurgical, endoscopic and radiological perspective. Minim Invasive Neurosurg 2005; 48: 218-223.

3. Beumer, D., Delwel, E.J., Kleinrensink, G.J., Akouri, S., Torres, A., Krisht, A.F.: The perforator-free zone of the posterior communicating artery and its relevance in approaches to the interpeduncular cistern, especially the transcavernous approach: an anatomic study. Neurosurgery 2007; 61 (Suppl 2): $187-191$.

4. Coupe, N.J., Athwal, R.K., Marshman, L.A., Brydon, H.L.: Subarachnoid hemorrhage emanating from a ruptured infundibulum: case report and literature review. Surg Neurol. 2007; 67: 204-206.

5. Cowan, J.A., Barkhoudarian, G., Yang, L.J.S., Thompson, B.G.: Progression of a posterior communicating artery infundibulum into an aneurysm in a patient with Alagille syndrome. J Neurosurg 2004; 101: 694-696.

6. Ebina, K., Ohkuma, H., Iwabuchi, T.: An angiographic study of incidence and morphology of infundibular dilatation of the posterior communicating artery. Neuroradiology 1986; 28: 23-29.

7. Endo, S., Furuichi, S., Takaba, M., Hirashima, Y., Nishijima, M., Takaku, A.: Clinical study of enlarged infundibular dilation of the origin of the posterior communicating artery. $\mathrm{J}$ Neurosurg 1995; 83: 421-425.

8. Epstein, F., Ransohoff, J., Budzilovich, G.: The clinical significance of junctional dilatation of the posterior communicating artery. J Neurosurg 1970; 33: 529-531.

9. Fox, J.L., Baiz, T.C., Jakoby, R.K,: Differentiation of aneurysm from infundibulum of the posterior communicating artery. J Neurosurg 1964; 21: 135-138.

10. González-Darder, J.M., Feliu, R., Pesudo, J.V., et al: Tratamiento quirúrgico de los aneurismas de la arteria comunicante posterior basado en el estudio de angio-tac con reconstrucción tridimensional y sin angiografía preoperatoría. Neurocirugía 2003; 14: 207-215.

11. Hassler, O., Saltzmann, G.F.: Angiographic and histologic changes in infundibular widening of the posterior communicating artery. Acta Radiol Diagn (Stockh) 1963; 1: 321-327.

12. Jennet, B., Bond, M.: Assessment of outcome after severe brain damage. A practical scale. Lancet 1975; 1: 480484.

13. Kaufmann, T.J., Razack, N., Cloft, H.J., Kallmes, D.F.: Dimpled appearance of a posterior communicating artery saccule: an angiographic indicator of arterial infundibula. AJR 2005; 185: 1358-1360

14. Kuwahara, S., Uga, S., Mori, K.: Successful treatment of a ruptured enlarged infundibular widening of the posterior 
communicating artery--case report. Neurol Med Chir 2001; 41: $25-28$.

15. Locksley, H.B.: Report on the co-operative study of intracranial aneurysms and subarachnoid hemorrhage: Section V-Part II: Natural history of subarachnoid hemorrhage, intracranial aneurysms and arteriovenous malformations-Based on 6368 cases in the co-operative study. J Neurosurg 1966; 25 : 321-368.

16. Marshman, L.A.G., Ward, P.J., Walter, P.H., Dossetor, R.S.: The progression of an infundibulum to aneurysm formation and rupture: case report and literature review Neurosurgery 1998; 43: 1445-1448.

17. Martins, C., Macanovic, M., Costa e Silva, I.E., Griz, F., Azevedo-Filho, H.R.C.: Progresión of an arterial infundibulum to aneurysm. Case report. Arq Neuropsiquiatr 2002; 60: 478-480.

18. Ohyama, T., Ohara, S., Momma, F.: Fatal subarachnoid hemorrhage due to ruptured infundibular widening of the posterior communicating artery: case report. Neurol Med Chir 1994; 34: 172-175.

19. Patrick, D., Appleby, A.: Infundibular widening of the posterior communicating artery progressing to true aneurysm. Br J Radiol 1983; 56: 59-60.

20. Radulovic, D., Nestorovic, B., Rakic, M., Janosevic, V.: Enlargement to a saccular aneurysm and subsequent rupture of infundibular widening of posterior communicating artery. Neurochirurgie 2006;52:525-528.

21. Report of the World Federation of Neurological Surgeons Committee on a Universal SAH Grading Scale.: J. Neurosurg 1998; 68: 985-986.

22. Rhoton, A.L. Jr.: The supratentorial arteries. Neurosurgery 51 (4 Suppl): S53-120, 2002.

23. Satoh, T., Omi, M., Ohsako, C., et al.: Differential diagnosis of the infundibular dilation and aneurysm of inter- nal carotid artery: assessment with fusion imaging of 3D MR cisternography/angiography. AJNR Am J Neuroradiol 2006; 27: 306-312.

24. Suzuki, K., Kodama, N., Sasaki, T., et al.: Confirmation of blood flow in perforating arteries using fluorescein cerebral angiography during aneurysm surgery. J Neurosurg 2007; 107: 68-73.

25. Taveras, J., Wood, E.: Diagnóstico Neuro-radiológico. Buenos Aires, Editorial Médica Panamericana, 1978.

26. Tomandl, B.F., Köstner, N.C., Schempershofe, M., et al.: CT angiography of intracranial aneurysms: a focus o postprocessing. RadioGraphics 2004; 24: 637-655

27. Wollschlaeger, G., Wollschlaeger, P.B., Lucas, F.V., López, V.F.: Experience and results with post-mortem cerebral angiography performed as routine procedure of the autopsy. Am J Roentgenol Radium Ther Nucl Med 1967; 101: 68-87.

28. Yamakawa, H., Sakai, N., Takenaka, K., et al.: Clinical análisis of recurrent subarachnoid hemorrhage after neck clipping surgery. Neurol Med Chir (Tokyo) 1997; 37: 380-386.

29. Yasargil, M.G.: Microneurosurgery, vol 1, New York; Thieme-Stratton, 1984.

30. Zager, E.L., Hackney, D.B.: Neurosurgical Forum: Clipping infundibula. J Neurosurg 1996; 84: 538-539

González-Darder, J.M.; Verdú-López, F,; Quilis-Quesada, V.: Manejo y tratamiento microquirúrgico de las dilataciones infundibulares de la arteria comunicante posterior. Serie de nueve casos y revisión de la literatura. Neurocirugía 2011; 22: 301-309.

Correspondencia: Dr. José M González-Darder. Servicio de Neurocirugía. Hospital Clínico Universitario. Avda. Blasco Ibáñez, 17. 46010-Valencia gonzalez_jos@gva.es 